\title{
The PBW Filtration, Demazure Modules and Toroidal Current Algebras
}

Evgeny FEIGIN †๋

$\dagger$ Mathematical Institute, University of Cologne, Weyertal 86-90, D-50931, Cologne, Germany

$\ddagger$ I.E. Tamm Department of Theoretical Physics, Lebedev Physics Institute, Leninski Prospect 53, Moscow, 119991, Russia

E-mail: evgfeig@gmail.com

Received July 04, 2008, in final form October 06, 2008; Published online October 14, 2008

Original article is available at http://www.emis.de/journals/SIGMA/2008/070/

\begin{abstract}
Let $L$ be the basic (level one vacuum) representation of the affine Kac-Moody Lie algebra $\widehat{\mathfrak{g}}$. The $m$-th space $F_{m}$ of the PBW filtration on $L$ is a linear span of vectors of the form $x_{1} \cdots x_{l} v_{0}$, where $l \leq m, x_{i} \in \widehat{\mathfrak{g}}$ and $v_{0}$ is a highest weight vector of $L$. In this paper we give two descriptions of the associated graded space $L^{\text {gr }}$ with respect to the PBW filtration. The "top-down" description deals with a structure of $L^{\mathrm{gr}}$ as a representation of the abelianized algebra of generating operators. We prove that the ideal of relations is generated by the coefficients of the squared field $e_{\theta}(z)^{2}$, which corresponds to the longest root $\theta$. The "bottom-up" description deals with the structure of $L^{\text {gr }}$ as a representation of the current algebra $\mathfrak{g} \otimes \mathbb{C}[t]$. We prove that each quotient $F_{m} / F_{m-1}$ can be filtered by graded deformations of the tensor products of $m$ copies of $\mathfrak{g}$.
\end{abstract}

Key words: affine Kac-Moody algebras; integrable representations; Demazure modules

2000 Mathematics Subject Classification: 17B67

\section{Introduction}

Let $\mathfrak{g}$ be a finite-dimensional simple Lie algebra, $\widehat{\mathfrak{g}}$ be the corresponding affine Kac-Moody Lie algebra (see [21, 25]). Let $L$ be the basic representation of $\widehat{\mathfrak{g}}$, i.e. an irreducible level one module with a highest weight vector $v_{0}$ satisfying condition $(\mathfrak{g} \otimes \mathbb{C}[t]) \cdot v_{0}=0$. The PBW filtration $F_{\bullet}$ on the space $L$ is defined as follows:

$$
F_{0}=\mathbb{C} v_{0}, F_{m+1}=F_{m}+\operatorname{span}\left\{x \cdot w: x \in \widehat{\mathfrak{g}}, w \in F_{m}\right\} .
$$

This filtration was introduced in [13] as a tool of study of vertex operators acting on the space of Virasoro minimal models (see [8]). In this paper we study the associated graded space $L^{\text {gr }}=F_{0} \oplus F_{1} / F_{0} \oplus \cdots$. We describe the space $L^{\text {gr }}$ from two different points of view: via "top-down" and "bottom-up" operators (the terminology of [24]).

On one hand, the space $L^{\text {gr }}$ is a module over the Abelian Lie algebra $\mathfrak{g}^{a b} \otimes t^{-1} \mathbb{C}\left[t^{-1}\right]$, where $\mathfrak{g}^{a b}$ is an Abelian Lie algebra whose underlying vector space is $\mathfrak{g}$. The module structure is induced from the action of the algebra of generating "top-down" operators $\mathfrak{g} \otimes t^{-1} \mathbb{C}\left[t^{-1}\right]$ on $L$. Thus $L^{\text {gr }}$ can be identified with a polynomial ring on the space $\mathfrak{g}^{a b} \otimes t^{-1} \mathbb{C}\left[t^{-1}\right]$ modulo certain ideal. Our first goal is to describe this ideal explicitly.

On the other hand, all spaces $F_{m}$ are stable with respect to the action of the subalgebra of annihilating operators $\mathfrak{g} \otimes \mathbb{C}[t]$ (the "bottom-up" operators). This gives $\mathfrak{g} \otimes \mathbb{C}[t]$ module structure

\footnotetext{
${ }^{\star}$ This paper is a contribution to the Special Issue on Kac-Moody Algebras and Applications. The full collection is available at http://www.emis.de/journals/SIGMA/Kac-Moody_algebras.html
} 
on each quotient $F_{m} / F_{m-1}$. Our second goal is to study these modules. We briefly formulate our results below.

For $x \in \mathfrak{g}$ let $x(z)=\sum_{i<0}\left(x \otimes t^{i}\right) z^{-i}$ be a generating function of the elements $x \otimes t^{i} \in \widehat{\mathfrak{g}}$, $i<0$. These series are also called fields. They play a crucial role in the theory of vertex operator algebras (see $[15,22,3]$ ). We will need the field which corresponds to the highest root $\theta$ of $\mathfrak{g}$. Namely, let $e_{\theta} \in \mathfrak{g}$ be a highest weight vector in the adjoint representation. It is well known (see for instance [3]) that the coefficients of $e_{\theta}(z)^{2}$ vanish on $L$. It follows immediately that the same relation holds on $L^{\mathrm{gr}}$. We note also that the Lie algebra $\mathfrak{g} \simeq \mathfrak{g} \otimes 1$ acts naturally on $L^{\mathrm{gr}}$. The following theorem is one of the central results of our paper.

Theorem 1. $L^{\mathrm{gr}}$ is isomorphic to the quotient of the universal enveloping algebra $\mathrm{U}\left(\mathfrak{g}^{a b} \otimes\right.$ $\left.t^{-1} \mathbb{C}\left[t^{-1}\right]\right)$ by the ideal $I$, which is the minimal $\mathfrak{g} \otimes 1$ invariant ideal containing all coefficients of the series $e_{\theta}(z)^{2}$.

This proves the level one case of the conjecture from [10]. We note that for $\mathfrak{g}=\mathfrak{s l}_{2}$ this theorem was proved in [13]. The generalization of this theorem for higher levels and $\mathfrak{g}=\mathfrak{s l}_{2}$ is conjectured in [10].

In order to prove this theorem and to make a connection with the "bottom-up" description we study the intersection of the PBW filtration with certain Demazure modules inside $L$. Recall (see [7]) that by definition a Demazure module $D(\lambda) \hookrightarrow L$ is generated by extremal vector of the weight $\lambda$ with an action of the universal enveloping algebra of the Borel subalgebra of $\widehat{\mathfrak{g}}$. We will only need the Demazure modules $D(N \theta)$. These modules are invariant with respect to the current algebra $\mathfrak{g} \otimes \mathbb{C}[t]$ and provide a filtration on $L$ by finite-dimensional spaces: $D(\theta) \hookrightarrow D(2 \theta) \hookrightarrow \cdots=L$ (see [18, 19]; some special cases are also contained in [12, 23]). Let $F_{m}(N)=D(N \theta) \cap F_{m}$ be an intersection of the Demazure module with the $m$-th space of the PBW filtration. This gives a filtration on $D(N \theta)$. In order to describe the filtration $F_{\bullet}(N)$ we use a notion of the fusion product of $\mathfrak{g} \otimes \mathbb{C}[t]$ modules (see $[17,11]$ ) and the Fourier-Littelmann results [19].

We recall that there exist two versions of the fusion procedure for modules over the current algebras. The first version constructs a graded $\mathfrak{g} \otimes \mathbb{C}[t]$ module $V_{1} * \cdots * V_{N}$ starting from the tensor product of cyclic $\mathfrak{g} \otimes \mathbb{C}[t]$ modules $V_{i}$. The other version also produces a graded $\mathfrak{g} \otimes \mathbb{C}[t]$ module $V_{1} * * \cdots * * V_{N}$, but in this case all $V_{i}$ are cyclic $\mathfrak{g}$ modules. (We note that second version is a special case of the first one). The fusion modules provide a useful tool for the study of the representation theory of current and affine algebras (see $[1,2,4,16,2,23,9,10,14,19]$ ). In particular, Fourier and Littelmann proved that there exists an isomorphism of $\mathfrak{g} \otimes \mathbb{C}[t]$ modules $D(N \theta) \simeq$ $D(\theta) * \cdots * D(\theta)$ ( $N$ times). Using this theorem and the $* *$-version of the fusion procedure, we endow the space $D(N \theta)$ with a structure of the representation of the toroidal current algebra $\mathfrak{g} \otimes \mathbb{C}[t, u]$ (see $[20,26]$ and references therein for some details on the representation theory of the toroidal algebras). This allows to prove our second main theorem:

Theorem 2. The $\mathfrak{g} \otimes \mathbb{C}[t]$ module $F_{m}(N) / F_{m-1}(N)$ can be filtered by $\left(\begin{array}{l}N \\ m\end{array}\right)$ copies of the $m$-th fusion power of the adjoint representation of $\mathfrak{g}$. In particular, $\operatorname{dim} F_{m}(N) / F_{m-1}(N)=$ $\left(\begin{array}{l}N \\ m\end{array}\right)(\operatorname{dim} \mathfrak{g})^{m}$.

The paper is organized as follows. In Section 2 we give the definition of the PBW filtration and of the induced filtration on Demazure modules. In Section 3 we study tensor products of cyclic $\mathfrak{g} \otimes \mathbb{C}[t]$ modules endowed with a structure of representations of toroidal algebra. In particular, we show that fusion product $D(1)^{* * N}$ is well defined. In Section 4 the results of Section 3 are applied to the module $D(N)$. We prove a graded version of the inequality $\operatorname{dim} F_{m}(N) / F_{m-1}(N) \geq\left(\begin{array}{l}N \\ m\end{array}\right)(\operatorname{dim} \mathfrak{g})^{m}$. In Section 5 the functional realization of the dual space $\left(L^{\mathrm{gr}}\right)^{*}$ is given. In Section 6 we combine all results of the previous sections and prove Theorems 1 and 2. We finish the paper with a list of the main notations. 


\section{The PBW filtration}

In this section we recall the definition and basic properties of the PBW filtration (see [13]).

Let $\mathfrak{g}$ be a simple Lie algebra, $\widehat{\mathfrak{g}}$ be the corresponding affine Kac-Moody Lie algebra:

$$
\widehat{\mathfrak{g}}=\mathfrak{g} \otimes \mathbb{C}\left[t, t^{-1}\right] \oplus \mathbb{C} K \oplus \mathbb{C} d .
$$

Here $K$ is a central element, $d$ is a degree element $\left(\left[d, x \otimes t^{i}\right]=-i x \otimes t^{i}\right)$ and

$$
\left[x \otimes t^{i}, y \otimes t^{j}\right]=[x, y] \otimes t^{i+j}+i \delta_{i+j, 0}(x, y) K,
$$

$x, y \in \mathfrak{g},(\cdot, \cdot)$ is a Killing form. Let $L$ be the basic representation of the affine Lie algebra, i.e. level one highest weight irreducible module with a highest weight vector $v_{0}$ satisfying

$$
(\mathfrak{g} \otimes \mathbb{C}[t]) \cdot v_{0}=0, \quad K v_{0}=v_{0}, \quad d v_{0}=0, \quad \mathrm{U}\left(\mathfrak{g} \otimes t^{-1} \mathbb{C}\left[t^{-1}\right]\right) \cdot v_{0}=L .
$$

The operator $d \in \widehat{\mathfrak{g}}$ defines a graded character of any subspace $V \hookrightarrow L$ by the formula

$$
\operatorname{ch}_{q} V=\sum_{n \geq 0} q^{n} \operatorname{dim}\{v \in V: d v=n v\} .
$$

For $x \in \mathfrak{g}$ we introduce a generating function $x(z)=\sum_{i>0}\left(x \otimes t^{-i}\right) z^{i}$ of the elements $x \otimes t^{i}$, $i<0$. We will mainly deal with the function $e_{\theta}(z)$, where $\theta$ is the highest weight of $\mathfrak{g}$ and $e_{\theta} \in \mathfrak{g}$ is a highest weight element. All coefficients

$$
\sum_{\substack{i+j=n \\ i, j \leq-1}}\left(e_{\theta} \otimes t^{i}\right)\left(e_{\theta} \otimes t^{j}\right)
$$

of the square of the series $e_{\theta}(z)$ are known to vanish on $L$ (this follows from the vertex operator realization of $L[15$, Theorem $A]$ ). Equivalently, $e_{\theta}(z)^{2}=0$ on $L$.

In what follows we will need a certain embedding of the basic $\widehat{\mathfrak{s l}_{2}}$ module into $L$. Namely, let $\mathfrak{s l}_{2}^{\theta}$ be a Lie algebra spanned by a $\mathfrak{s l}_{2}$-triple $e_{\theta}, f_{\theta}$ and $h_{\theta}$, where $e_{\theta}$ and $f_{\theta}$ are highest and lowest weight vectors in the adjoint representation of $\mathfrak{g}$. Then the restriction map defines a structure of $\widehat{\mathfrak{s l}_{2}^{\theta}}$ module on $L$. In particular, the space $\mathrm{U}\left(\widehat{\mathfrak{s l}_{2}^{\theta}}\right) \cdot v_{0}$ is isomorphic to the basic representation of $\widehat{\mathfrak{s l}_{2}}$, since the defining relations $\left(e_{\theta} \otimes t^{-1}\right)^{2} v_{0}=0$ and $\left(\mathfrak{s l}_{2}^{\theta} \otimes 1\right) v_{0}=0$ are satisfied (see [25, Lemma 2.1.7]).

We now define the PBW filtration $F$ • on $L$. Namely, let

$$
F_{0}=\mathbb{C} v_{0}, \quad F_{m+1}=F_{m}+\operatorname{span}\left\{\left(x \otimes t^{-i}\right) w, x \in \mathfrak{g}, i>0, w \in F_{m}\right\} .
$$

Then $F_{\bullet}$ is an increasing filtration converging to $L$. We denote the associated graded space by $L^{\mathrm{gr}}$ :

$$
L^{\mathrm{gr}}=\bigoplus_{m \geq 0} L_{m}^{\mathrm{gr}}, \quad L_{m}^{\mathrm{gr}}=\mathrm{gr}_{m} F_{\bullet}=F_{m} / F_{m-1}
$$

In what follows we denote by $\mathfrak{g}^{a b}$ an Abelian Lie algebra with the underlying vector space isomorphic to $\mathfrak{g}$. We endow $\mathfrak{g}^{a b}$ with a structure of $\mathfrak{g}$ module via the adjoint action of $\mathfrak{g}$ on itself.

Lemma 1. The action of $\mathfrak{g} \otimes \mathbb{C}[t]$ on $L$ induces an action of the same algebra on $L^{\mathrm{gr}}$. The action of $\mathfrak{g} \otimes t^{-1} \mathbb{C}\left[t^{-1}\right]$ on $L$ induces an action of the algebra $\mathfrak{g}^{a b} \otimes t^{-1} \mathbb{C}\left[t^{-1}\right]$ on $L^{\mathrm{gr}}$. 
Proof. All spaces $F_{m}$ are invariant with respect to the action of $\mathfrak{g} \otimes \mathbb{C}[t]$, since the condition $(\mathfrak{g} \otimes \mathbb{C}[t]) \cdot v_{0}=0$ is satisfied. Hence we obtain an induced action on the quotient spaces $F_{m} / F_{m-1}$.

Operators $x \otimes t^{i}, i<0$ do not preserve $F_{m}$ but map it to $F_{m+1}$. Therefore, each element $x \otimes t^{i}, i<0$ produce an operator acting from $L_{m}^{\mathrm{gr}}$ to $L_{m+1}^{\mathrm{gr}}$. An important property of these operators on $L^{\mathrm{gr}}$ is that they mutually commute, since the composition $\left(x \otimes t^{i}\right)\left(y \otimes t^{j}\right)$ acts from $F_{m}$ to $F_{m+2}$ but the commutator $\left[x \otimes t^{i}, y \otimes t^{j}\right]=[x, y] \otimes t^{i+j}$ maps $F_{m}$ to $F_{m+1}$. Lemma is proved.

The goal of our paper is to describe the structure of $L^{\text {gr }}$ as a representation of $\mathfrak{g} \otimes \mathbb{C}[t]$ and of $\mathfrak{g}^{a b} \otimes t^{-1} \mathbb{C}\left[t^{-1}\right]$. It turns out that these two structures are closely related.

Lemma 2. Let $I \hookrightarrow \mathrm{U}\left(\mathfrak{g}^{a b} \otimes t^{-1} \mathbb{C}\left[t^{-1}\right]\right)$ be the minimal $\mathfrak{g}$-invariant ideal containing all coeffcients of the series $e_{\theta}(z)^{2}$. Then there exists a surjective homomorphism

$$
\mathrm{U}\left(\mathfrak{g}^{a b} \otimes t^{-1} \mathbb{C}\left[t^{-1}\right]\right) / I \rightarrow L^{\mathrm{gr}}
$$

of $\mathfrak{g}^{a b} \otimes t^{-1} \mathbb{C}\left[t^{-1}\right]$ modules mapping 1 to $v_{0}$.

Proof. Follows from the relation $e_{\theta}(z)^{2} v_{0}=0$.

One of our goals is to prove that the homomorphism (1) is an isomorphism.

Recall (see [18]) that $L$ is filtered by finite-dimensional Demazure modules [7]. A Demazure module $D$ is generated by an extremal vector with the action of the algebra of generating operators. We will only need special class of Demazure modules. Namely, for $N \geq 0$ let $v_{N} \in L$ be the vector of weight $N \theta$ defined by

$$
v_{N}=\left(e_{\theta} \otimes t^{-N}\right)^{N} v_{0} .
$$

We recall that $N \theta$ is an extremal weight for $L$ and thus $v_{N}$ spans weight $N \theta$ subspace of $L$. Let $D(N) \hookrightarrow L$ be the Demazure module generated by vector $v_{N}$. Thus $D(N)$ is cyclic $\mathfrak{g} \otimes \mathbb{C}[t]$ module with cyclic vector $v_{N}$. It is known (see $[25,18]$ ) that these modules are embedded into each other and the limit coincides with $L$ :

$$
D(1) \hookrightarrow D(2) \hookrightarrow \cdots=L
$$

We introduce an induced PBW filtration on $D(N)$ :

$$
F_{0}(N) \hookrightarrow F_{1}(N) \hookrightarrow \cdots=D(N), \quad F_{m}(N)=D(N) \cap F_{m}
$$

Obviously, each space $F_{m}(N)$ is $\mathfrak{g} \otimes \mathbb{C}[t]$ invariant.

Lemma 3. $F_{N}(N)=D(N)$, but $F_{N-1}(N) \neq D(N)$.

Proof. First equality holds since $\left(e_{\theta} \otimes t^{-N}\right)^{N} v_{0}=v_{N}$ and $D(N)$ is cyclic. In addition $v_{N} \notin F_{N-1}$ because all weights of $F_{m}$ (as a representation of $\mathfrak{g} \simeq \mathfrak{g} \otimes 1$ ) are less than or equal to $m \theta$.

Consider the associated graded object

$$
\operatorname{gr} F(N)=\bigoplus_{m=0}^{\infty} \operatorname{gr}_{m} F(N), \quad \operatorname{gr}_{m} F(N)=F_{m}(N) / F_{m-1}(N) .
$$

We note that each space $\operatorname{gr}_{m} F(N)$ has a natural structure of $\mathfrak{g} \otimes \mathbb{C}[t]$ module. 


\section{$3 \quad t^{N}$-filtration}

In this section we describe the filtration (2) using the generalization of the fusion product of $\mathfrak{g} \otimes \mathbb{C}[t]$ modules from [17] and a theorem of [19]. We first recall the definition of the fusion product of $\mathfrak{g} \otimes \mathbb{C}[t]$ modules.

Let $V$ be a $\mathfrak{g} \otimes \mathbb{C}[t]$ module, $c$ be a complex number. We denote by $V(c)$ a $\mathfrak{g} \otimes \mathbb{C}[t]$ module which coincides with $V$ as a vector space and the action is twisted by the Lie algebra homomorphism

$$
\phi(c): \mathfrak{g} \otimes \mathbb{C}[t] \rightarrow \mathfrak{g} \otimes \mathbb{C}[t], \quad x \otimes t^{k} \mapsto x \otimes(t+c)^{k} .
$$

Let $V_{1}, \ldots, V_{N}$ be cyclic representations of the current algebra $\mathfrak{g} \otimes \mathbb{C}[t]$ with cyclic vectors $v_{1}, \ldots, v_{N}$. Let $c_{1}, \ldots, c_{N}$ be a set of pairwise distinct complex numbers. The fusion product $V_{1}\left(c_{1}\right) * \cdots * V_{N}\left(c_{N}\right)$ is a graded deformation of the usual tensor product $V_{1}\left(c_{1}\right) \otimes \cdots \otimes V_{N}\left(c_{N}\right)$. More precisely, let $\mathrm{U}(\mathfrak{g} \otimes \mathbb{C}[t])_{s}$ be a natural grading on the universal enveloping algebra coming from the counting of the $t$ degree. Because of the condition $c_{i} \neq c_{j}$ for $i \neq j$, the tensor product $\bigotimes_{i=1}^{N} V_{i}\left(c_{i}\right)$ is a cyclic $\mathrm{U}(\mathfrak{g} \otimes \mathbb{C}[t])$ module with a cyclic vector $\otimes_{i=1}^{N} v_{i}$. Therefore, the grading on $\mathrm{U}(\mathfrak{g} \otimes \mathbb{C}[t])$ induces an increasing fusion filtration

$$
\mathrm{U}(\mathfrak{g} \otimes \mathbb{C}[t])_{\leq s} \cdot\left(v_{1} \otimes \cdots \otimes v_{N}\right)
$$

on the tensor product.

Definition 1. The fusion product $V_{1}\left(c_{1}\right) * \cdots * V_{N}\left(c_{N}\right)$ of $\mathfrak{g} \otimes \mathbb{C}[t]$ modules $V_{i}$ is an associated graded $\mathfrak{g} \otimes \mathbb{C}[t]$ module with respect to the filtration (3) on the tensor product $V_{1}\left(c_{1}\right) \otimes \cdots \otimes$ $V_{N}\left(c_{N}\right)$. We denote the $m$-th graded component by $\operatorname{gr}_{m}\left(V_{1}\left(c_{1}\right) * \cdots * V_{N}\left(c_{N}\right)\right)$.

We note that in many special cases the $\mathfrak{g} \otimes \mathbb{C}[t]$ module structure of the fusion product does not depend on the parameters $c_{i}$ (see for example $[1,5,11,19,16]$ ). We then omit the parameters $c_{i}$ and denote the corresponding fusion product simply by $V_{1} * \cdots * V_{N}$.

In what follows we will need a special but important case of the procedure described above. Namely, let $V_{i}$ be cyclic $\mathfrak{g}$ modules. One can extend the $\mathfrak{g}$ module structure to the $\mathfrak{g} \otimes \mathbb{C}[t]$ module structure by letting $\mathfrak{g} \otimes t \mathbb{C}[t]$ to act by zero. We denote the corresponding $\mathfrak{g} \otimes \mathbb{C}[t]$ modules by $\bar{V}_{i}$.

Definition 2. Let $V_{1}, \ldots, V_{N}$ be a set of cyclic $\mathfrak{g}$ modules. Then a $\mathfrak{g} \otimes \mathbb{C}[t]$ module $V_{1}\left(c_{1}\right) *$ $* \cdots * * V_{N}\left(c_{N}\right)$ is defined by the formula:

$$
V_{1}\left(c_{1}\right) * * \cdots * * V_{N}\left(c_{N}\right)=\bar{V}_{1}\left(c_{1}\right) * \cdots * \bar{V}_{N}\left(c_{N}\right) .
$$

We denote the $m$-th graded component by $\operatorname{gr}_{m}\left(V_{1}\left(c_{1}\right) * * \cdots * * V_{N}\left(c_{N}\right)\right)$.

Remark 1. The fusion procedure described in Definition 2 can be reformulated as follows. One starts with a tensor product of evaluation $\mathfrak{g} \otimes \mathbb{C}[t]$ modules $V_{i}\left(c_{i}\right)$, where $x \otimes t^{k}$ acts on $V_{i}$ by $c_{i}^{k} x$ (we evaluate $t$ at the point $c_{i}$ ). Then one constructs the fusion filtration and associated graded module (according to Definition 1).

Remark 2. Let us stress the main difference between Definitions 1 and 2. Definition 1 gets as an input a set of cyclic representations of the current algebra $\mathfrak{g} \otimes \mathbb{C}[t]$ and as a result produces a $t$-graded $\mathfrak{g} \otimes \mathbb{C}[t]$ module. The input of Definition 2 is a set of cyclic $\mathfrak{g}$ modules and an output is again a $t$-graded $\mathfrak{g} \otimes \mathbb{C}[t]$ module.

We now recall a theorem from [19] which uses the fusion procedure to construct the Demazure module $D(N)$ starting from $D(1)$. 
Theorem 3 ([19]). The $N$-th fusion power $D(1)^{* N}$ is independent on the evaluation parameters $c_{i}$. The $\mathfrak{g} \otimes \mathbb{C}[t]$ modules $D(1)^{* N}$ and $D(N)$ are isomorphic.

We recall that as a $\mathfrak{g}$ module $D(1)$ is isomorphic to the direct sum of trivial and adjoint representations, $D(1) \simeq \mathbb{C} \oplus \mathfrak{g}$. The trivial representation is annihilated by $\mathfrak{g} \otimes \mathbb{C}[t]$, the adjoint representation is annihilated by $\mathfrak{g} \otimes t^{2} \mathbb{C}[t]$ and $\mathfrak{g} \otimes \mathbb{C}[t]$ maps $\mathfrak{g}$ to $\mathbb{C}$.

Our idea is to combine the theorem above and Definition 2 with $\mathfrak{g}$ being the current algebra $\mathfrak{g} \otimes \mathbb{C}[u]$ (see also [4], where Definition 2 is used in affine settings). Definition 2 works for arbitrary $\mathfrak{g}$ and produces a representation of an algebra with an additional current variable. In particular, starting from the $\mathfrak{g} \otimes \mathbb{C}[u]$ modules $V_{i}=D(1)$ and an $N$ tuple of pairwise distinct complex numbers $c_{1}, \ldots, c_{N}$ one gets a new bi-graded $\mathfrak{g} \otimes \mathbb{C}[t, u]$ module. The resulting module can be obtained from the Demazure module $D(N)$ by a rather simple procedure which we are going to describe now.

Let $V_{1}, \ldots, V_{N}$ be cyclic representations of the algebra $\mathfrak{g} \otimes \mathbb{C}[t] /\left\langle t^{2}\right\rangle$. Hence $V_{1}\left(c_{1}\right) * \cdots * V_{N}\left(c_{N}\right)$ is a cyclic $\mathfrak{g} \otimes \mathbb{C}[t] /\left\langle t^{2 N}\right\rangle$ module. We consider a decreasing filtration $\mathrm{U}(\mathfrak{g} \otimes \mathbb{C}[t])^{j}$ on the universal enveloping algebra defined by

$$
\mathrm{U}(\mathfrak{g} \otimes \mathbb{C}[t])^{0}=\mathrm{U}(\mathfrak{g} \otimes \mathbb{C}[t]), \quad \mathrm{U}(\mathfrak{g} \otimes \mathbb{C}[t])^{j+1}=\left(\mathfrak{g} \otimes t^{N} \mathbb{C}[t]\right) \mathrm{U}(\mathfrak{g} \otimes \mathbb{C}[t])^{j} .
$$

This filtration induces a decreasing filtration $G^{j}$ on the fusion product $V_{1}\left(c_{1}\right) * \cdots * V_{N}\left(c_{N}\right)$ (since it is cyclic $\mathrm{U}(\mathfrak{g} \otimes \mathbb{C}[t])$ module). $G^{\bullet}$ will be also referred to as a $t^{N}$-filtration. Consider the associated graded space

$$
\operatorname{gr} G^{\bullet}=\bigoplus_{j=0}^{N} \operatorname{gr}^{j} G^{\bullet}, \quad \operatorname{gr}^{j} G^{\bullet}=G^{j} / G^{j+1}
$$

Since each space $G^{j}$ is $\mathfrak{g} \otimes \mathbb{C}[t]$ invariant one gets a structure of $\mathfrak{g} \otimes \mathbb{C}[t] /\left\langle t^{N}\right\rangle$ module on each space $G^{j} / G^{j+1}$. In addition an element from $\mathfrak{g} \otimes t^{N} \mathbb{C}[t] /\left\langle t^{2 N}\right\rangle$ produces a degree 1 operator on (5) mapping $\operatorname{gr}^{j} G^{\bullet}$ to $\operatorname{gr}^{j+1} G^{\bullet}$. We thus obtain a structure of $\mathfrak{g} \otimes \mathbb{C}[t, u] /\left\langle t^{N}, u^{2}\right\rangle$ module on (5), where $\mathfrak{g} \otimes u \mathbb{C}[t]$ denotes an algebra of degree one operators on gr $G \bullet$ coming from the action of $\mathfrak{g} \otimes t^{N} \mathbb{C}[t] /\left\langle t^{2 N}\right\rangle$.

On the other hand let us consider the modules $V_{i}$ as representations of the Lie algebra $\mathfrak{g} \otimes \mathbb{C}[u] /\left\langle u^{2}\right\rangle$ (simply replacing $t$ by $u$ ). We denote these modules as $V_{i}^{u}$. Then the bi-graded tensor product $V_{1}^{u}\left(c_{1}\right) * * \cdots * * V_{N}^{u}\left(c_{N}\right)$ is a representation of the Lie algebra $\mathfrak{g} \otimes \mathbb{C}[t, u] /\left\langle t^{N}, u^{2}\right\rangle$.

Proposition 1. We have an isomorphism of $\mathfrak{g} \otimes \mathbb{C}[t, u] /\left\langle t^{N}, u^{2}\right\rangle$ modules

$$
\operatorname{gr} G^{\bullet} \simeq V_{1}^{u}\left(c_{1}\right) * * \cdots * * V_{N}^{u}\left(c_{N}\right) .
$$

Proof. The idea of the proof is as follows. We start with the tensor product $V_{1} \otimes \cdots \otimes V_{N}$ and apply the fusion filtration. Afterwards we apply the $t^{N}$-filtration $G^{\bullet}$. Combining these operations with certain changes of basis of current algebra we arrive at the definition of the bi-graded module $V_{1}^{u} * * \cdots * * V_{N}^{u}$. We give details below.

For an element $x \otimes t^{i} \in \mathfrak{g} \otimes \mathbb{C}[t]$ let $\left(x \otimes t^{i}\right)^{(j)}$ be the operator on the tensor product $V_{1} \otimes \cdots \otimes V_{N}$ defined by

$$
\left(x \otimes t^{i}\right)^{(j)}=\mathrm{Id}^{\otimes j-1} \otimes\left(x \otimes t^{i}\right) \otimes \mathrm{Id}^{\otimes N-j},
$$

i.e. $\left(x \otimes t^{i}\right)^{(j)}$ acts as $x \otimes t^{i}$ on $V_{j}$ and as an identity operator on the other factors. In order to construct the fusion product one starts with the operators

$$
A\left(x \otimes t^{i}\right)=\sum_{j=1}^{N}\left(x \otimes\left(t+c_{j}\right)^{i}\right)^{(j)}
$$


where $c_{j}$ are pairwise distinct numbers. These operators define an action of the algebra $\mathfrak{g} \otimes \mathbb{C}[t]$ on the tensor product $V_{1}\left(c_{1}\right) \otimes \cdots \otimes V_{N}\left(c_{N}\right)$. Since $x \otimes t^{i}$ with $i>1$ vanish on $V_{j}$ we obtain

$$
A\left(x \otimes t^{i}\right)=\sum_{j=1}^{N} c_{j}^{i}(x \otimes 1)^{(j)}+\sum_{j=1}^{N} i c_{j}^{i-1}(x \otimes t)^{(j)} .
$$

The next step is to pass to the associated graded module with respect to the fusion filtration. By definition, operators of the form

$$
A\left(x \otimes t^{k}\right)+\text { linear combination of } A\left(x \otimes t^{l}\right), \quad l<k
$$

act on the associated graded module in the same way as $A\left(x \otimes t^{k}\right)$ (the lower degree term vanish after passing to the associated graded space). We are going to fix special linear changes in (8) for $N \leq k<2 N$ which make the expressions for $A\left(x \otimes t^{k}\right)$ simpler.

Let $\alpha_{0}, \alpha_{1}, \ldots, \alpha_{N-1}$ be numbers such that for all $1 \leq j \leq N$

$$
c_{j}^{N}+\sum_{i=0}^{N-1} \alpha_{i} c_{j}^{i}=0
$$

We state that

$$
A\left(x \otimes t^{N+s}\right)+\sum_{i=0}^{N-1} \alpha_{i} A\left(x \otimes t^{s+i}\right)=\sum_{j=1}^{N} c_{j}^{s}(x \otimes t)^{(j)} \prod_{k \neq j}\left(c_{j}-c_{k}\right)
$$

for all $0 \leq s \leq N-1$. Let

$$
f(x)=x^{N}+\alpha_{N-1} x^{N-1}+\cdots+\alpha_{0} .
$$

Then $f(x)=\prod_{k=1}^{N}\left(x-c_{k}\right)$. Therefore, for the derivative $c_{j}^{s} f^{\prime}\left(c_{j}\right)$ one gets

$$
c_{j}^{s} f^{\prime}\left(c_{j}\right)=N c_{j}^{N+s-1}+\sum_{i=0}^{N-1} i \alpha_{i} c_{j}^{i+s-1}=c_{j}^{s} \prod_{k \neq j}\left(c_{j}-c_{k}\right) .
$$

This proves (9).

Using formula (9), we replace operators $A\left(x \otimes t^{i}\right), 0 \leq i<2 N$ by operators $B\left(x \otimes t^{i}\right)$ as follows

$$
\begin{aligned}
& B\left(x \otimes t^{i}\right)=\sum_{j=1}^{N} c_{j}^{i}(x \otimes 1)^{(j)}+\sum_{j=1}^{N} i c_{j}^{i-1}(x \otimes t)^{(j)}, \quad 0 \leq i<N, \\
& B\left(x \otimes t^{N+i}\right)=\sum_{i=1}^{N} c_{i}^{s}(x \otimes t)^{(i)} \prod_{k \neq j}\left(c_{j}-c_{k}\right), \quad 0 \leq i<N,
\end{aligned}
$$

thus performing the linear change (8). So we redefine half of the operators $A\left(x \otimes t^{i}\right)$ and leave the other half unchanged.

In order to construct the left hand side of (6) one first applies the fusion filtration to the algebra of operators $B\left(x \otimes t^{i}\right)$ and afterwards proceeds with the $t^{N}$-filtration. The last step means that the subtraction of a linear combination of the operators $B\left(x \otimes t^{N+i}\right), 0 \leq i<N$ 
from $B\left(x \otimes t^{i}\right), 0 \leq i<N$ does not change the structure of the resulting module. Redefining the operators (10) we arrive at the following operators:

$$
\begin{aligned}
& C\left(x \otimes t^{i}\right)=\sum_{j=1}^{N} c_{j}^{i}(x \otimes 1)^{(j)}, \quad 0 \leq i<N, \\
& C\left(x \otimes t^{N+i}\right)=\sum_{i=j}^{N} c_{j}^{i}(x \otimes t)^{(j)}, \quad 0 \leq i<N .
\end{aligned}
$$

Note that we can remove constants $\prod_{k \neq j}\left(c_{j}-c_{k}\right)$ from $B\left(x \otimes t^{N+i}\right)$ since this procedure corresponds simply to rescaling the variable $t$ in each $V_{i}$.

Summarizing all the formulas above we arrive at the following two steps construction of the left hand side of (6):

- apply the fusion procedure to the operators $C\left(x \otimes t^{i}\right), 0 \leq i<2 N$,

- attach a $u$-degree 1 to each of the operators $C\left(x \otimes t^{N+i}\right), 0 \leq i<0$.

In order to construct the right hand side of (6) one uses the same procedure with $C\left(x \otimes t^{N+i}\right)$ being operators which correspond to $x \otimes u t^{i}$ (see (4) and (5)). Thus we have shown that the associated graded to the fusion product with respect to the $t^{N}$-filtration is isomorphic to the module $V_{1} * * \cdots * * V_{N}$. Proposition is proved.

Corollary 1. The fusion product $D(1) * * \cdots * * D(1)$ does not depend on the evaluation parameters.

Corollary 2. The fusion product of the adjoint representations

$$
\mathfrak{g}\left(c_{1}\right) * * \cdots * * \mathfrak{g}\left(c_{N}\right)
$$

is independent of the parameters $c_{1}, \ldots, c_{N}$.

Proof. By definition, the zeroth graded component with respect to the $t$ grading of the module

$$
D(1)\left(c_{1}\right) * * \cdots * * D(1)\left(c_{N}\right)
$$

is isomorphic to the fusion product $\mathfrak{g}\left(c_{1}\right) * * \cdots * * \mathfrak{g}\left(c_{N}\right)$. From the Proposition above we obtain an isomorphism

$$
\mathfrak{g}\left(c_{1}\right) * * \cdots * * \mathfrak{g}\left(c_{n}\right) \simeq D(N) /\left(\mathfrak{g} \otimes t^{N} \mathbb{C}[t]\right) D(N)
$$

for any $c_{1}, \ldots, c_{n}$. Thus the left hand side is independent of $c_{i}$.

We finish this section introducing an "energy" operator $\bar{d}$ on the fusion product. The operator $\bar{d}$ acts by a constant $m$ on the graded component

$$
\operatorname{gr}_{m}\left(V_{1}\left(c_{1}\right) * \cdots * V_{N}\left(c_{N}\right)\right) \text {. }
$$

The operator $\bar{d}$ defines a graded character of $V_{1}\left(c_{1}\right) * \cdots * V_{n}\left(c_{n}\right)$ by the standard formula formula

$$
\overline{\operatorname{ch}}_{q} V_{1}\left(c_{1}\right) * \cdots * V_{n}\left(c_{n}\right)=\sum_{n \geq 0} q^{n} \operatorname{dim}\{v: \bar{d} v=n v\} .
$$

An analogous formula defines a character of $V_{1}\left(c_{1}\right) * * \cdots * * V_{n}\left(c_{n}\right)$. We note that this grading has nothing to do with the Cartan grading coming from the action of the Cartan subalgebra. We do not consider the latter grading in this paper. 
Remark 3. Let $V_{i} \simeq D(1)$ for all $i$. Then the fusion module is independent on the evaluation parameters and $D(1)^{* N}$ is isomorphic to the Demazure module $D(N)$ (see [19]). By the very definition we have an embedding $D(N) \hookrightarrow L$. Thus both operators $d$ and $\bar{d}$ are acting on $D(N)$, satisfying the relations

$$
\left[d, x \otimes t^{i}\right]=-i x \otimes t^{i}, \quad\left[\bar{d}, x \otimes t^{i}\right]=i x \otimes t^{i} .
$$

Since $d v_{N}=N^{2} v_{N}$ and $\bar{d} v_{N}=0$ we have a simple identity $\bar{d}=N^{2}-d$.

\section{Demazure modules}

In this section we study the fusion filtration on the tensor product $D(1)^{\otimes N}$ and the induced PBW filtration on the Demazure modules $D(N)$. We also derive some connections between these filtrations.

Let $D^{u}(1)$ be the $\mathfrak{g} \otimes \mathbb{C}[u] /\left\langle u^{2}\right\rangle$ module obtained from $D(1)$ by substituting $u$ instead of $t$. In particular, $\left(D^{u}(1)\right)^{* * N}$ is a $(t, u)$ bi-graded representation of the algebra $\mathfrak{g} \otimes \mathbb{C}[t, u] /\left\langle t^{N}, u^{2}\right\rangle$. Here $t$-grading is exactly the fusion grading $\operatorname{gr}_{j} D^{u}(1) * \cdots * D^{u}(1)$ and the $u$-grading comes from the grading on $D^{u}(1)$, which assigns degree zero to $\mathfrak{g}$ and degree one to $\mathbb{C} v_{0}$. We consider the decomposition

$$
\left(D^{u}(1)\right)^{* * N}=\bigoplus_{m=0}^{N} W(m)
$$

into the graded components with respect to the $u$-grading. (The $u$-grading is bounded from above by $N$ since the $u$-grading in each of $D(1)$ could be either 0 or 1$)$. Note that each space $W(m)$ is a representation of $\mathfrak{g} \otimes \mathbb{C}[t]$. We want to show that $W(m)$ can be filtered by certain number of copies of the fusion product $\mathfrak{g}^{* * N-m}$. The precise statement is given in the following proposition.

Proposition 2. Let $D^{u}(1)^{* * N}$ be a bi-graded tensor product of $N$ copies of $\mathfrak{g} \otimes \mathbb{C}[u]$-module $D(1)$. Then

- For any $0 \leq m \leq N$ the $\mathfrak{g} \otimes \mathbb{C}[t]$ module $W(m)$ can be filtered by $\left(\begin{array}{l}N \\ m\end{array}\right)$ copies of the $\mathfrak{g} \otimes \mathbb{C}[t]$-module $\mathfrak{g}^{* * N-m}$.

- The cyclic vectors of the modules $\mathfrak{g}^{* * N-m}$ above are the images of the vectors

$$
\left(f^{\theta} \otimes u t^{i_{1}}\right) \cdots\left(f^{\theta} \otimes u t^{i_{m}}\right) v_{N}, \quad 0 \leq i_{1} \leq \cdots \leq i_{m} \leq N-m .
$$

Remark 4. We first give a non rigorous, but conceptual explanation of the statement of the proposition above. Recall that $D^{u}(1)$ is isomorphic to $\mathfrak{g} \oplus \mathbb{C}$ as a $\mathfrak{g}$ module. Let $v_{1}, v_{0} \in D(1)$ be highest weight vectors of $\mathfrak{g}$ and $\mathbb{C}$ respectively. Then $\left(f_{\theta} \otimes u\right) v_{1}=v_{0}$ and $\left(f_{\theta} \otimes u\right)^{2} v_{0}=0$. Therefore, after making the fusion $D^{u}(1)^{* * N}$, the tensor product of $N$ copies of 2-dimensional vector space $\operatorname{span}\left\{v_{0}, v_{1}\right\}$ will be deformed into the $N$-fold fusion product of two-dimensional representations of the algebra $\mathbb{C}\left[f_{\theta}\right]$. The set of vectors (11) represents a basis of this fusion product (see $[6,11]$ ). Hence $D^{u}(1)^{* N}$ is equal to the $\mathrm{U}(\mathfrak{g} \otimes \mathbb{C}[t])$ span of the vectors of the form (11). We now want to describe the space $\mathrm{U}(\mathfrak{g} \otimes \mathbb{C}[t]) \cdot w_{m}$, where $w_{m}$ is of the form (11) with exactly $m$ factors. Note that $w_{m}$ is a linear combination of the vectors of the form

$$
v_{i_{1}} \otimes \cdots \otimes v_{i_{N}}
$$

where $i_{\alpha}$ equal 0 or 1 and the number of $\alpha$ such that $i_{\alpha}=0$ is equal to $m$. This means that the space $\mathrm{U}(\mathfrak{g} \otimes \mathbb{C}[t]) \cdot w_{m}$ is embedded into the direct sum of $\left(\begin{array}{l}N \\ m\end{array}\right)$ copies of the tensor product $\mathfrak{g}^{\otimes m}$. 
Hence it is natural to expect that after passing to the associated graded object with respect to the fusion filtration one arrives at the fusion product $\mathfrak{g}^{* * m}$. This is not exactly true. In order to make the statement precise one additional filtration is needed (that is the reason why $W(m)$ is not the direct sum of the fusion products, but rather can be filtered by these modules).

We now give the proof of Proposition 2.

Proof. As a starting point we note the isomorphism of $\mathfrak{g} \otimes \mathbb{C}[t]$-modules

$$
W(0) \simeq \mathfrak{g}^{* * N}
$$

In fact, $D(1) \simeq \mathfrak{g} \oplus \mathbb{C} v_{0}$ with cyclic vector being the highest weight vector of $\mathfrak{g}$. The algebra $\mathfrak{g} \otimes u$ maps $\mathbb{C}$ to zero and $\mathfrak{g}$ to $\mathbb{C}$. In particular,

$$
\left(f_{\theta} \otimes u\right) \cdot v_{1}=v_{0}
$$

Hence if we do not apply operators with positive powers of $u$ (i.e. we consider the space $W(0)$ ) we arrive at the usual fusion product of $N$ copies of $\mathfrak{g}$.

We now introduce a decreasing filtration $W^{j}(m)$ such that the associated graded object is isomorphic to the direct sum of $\left(\begin{array}{l}N \\ m\end{array}\right)$ copies of $\mathfrak{g}^{* * m}$. Let

$$
w_{i_{1}, \ldots, i_{m}}=\left(f^{\theta} \otimes u t^{i_{1}}\right) \ldots\left(f^{\theta} \otimes u t^{i_{m}}\right) v_{N}, \quad 0 \leq i_{1} \leq \cdots \leq i_{m} \leq N-m .
$$

We set

$$
W^{n}(m)=\mathrm{U}(\mathfrak{g} \otimes \mathbb{C}[t]) \cdot \operatorname{span}\left\{w_{i_{1}, \ldots, i_{m}}: i_{1}+\cdots+i_{m} \geq n\right\} .
$$

In particular, $W^{0}(m)=W(m)$ and each space $W^{n}(m)$ is $\mathfrak{g} \otimes \mathbb{C}[t]$ invariant. We state that the associated graded space

$$
W^{0}(m) / W^{1}(m) \oplus W^{1}(m) / W^{2}(m) \oplus \cdots
$$

is isomorphic to the direct sum of $\left(\begin{array}{l}N \\ m\end{array}\right)$ copies of the modules $\mathfrak{g}^{* * N-m}$. Moreover the highest weight vectors of these modules are exactly the images of $w_{i_{1}, \ldots, i_{m}}$. We prove this statement for $m=1$. The proof for other $m$ is very similar.

For $m=1$ we have

$$
w_{i}=f_{\theta} \otimes u t^{i}=\sum_{j=1}^{N} c_{j}^{i} v_{1}^{\otimes j-1} \otimes v_{0} \otimes v_{1}^{\otimes N-j}, \quad i=0, \ldots, N-1 .
$$

We want to show that

$$
W^{i}(1) / W^{i+1}(1) \simeq \mathfrak{g}^{* * N-1}, \quad W^{i}(1)=\mathrm{U}(\mathfrak{g} \otimes \mathbb{C}[t]) \cdot \operatorname{span}\left\{w_{i}, \ldots, w_{N-1}\right\}
$$

Let $\alpha_{i, j}, 1 \leq i, j \leq N$ be some numbers. Denote

$$
\tilde{W}^{i}(1)=\mathrm{U}(\mathfrak{g} \otimes \mathbb{C}[t]) \cdot \operatorname{span}\left\{\sum_{j=1}^{N} \alpha_{i+1, j} w_{i}, \ldots, \sum_{j=1}^{N} \alpha_{N, j} w_{N-1}\right\} .
$$

We state that

$$
W^{i}(1) / W^{i+1}(1) \simeq \tilde{W}^{i}(1) / \tilde{W}^{i+1}(1)
$$


as $\mathfrak{g} \otimes \mathbb{C}[t]$ modules. In fact, adding to $w_{i}$ a linear combination of $w_{j}$ with $j<i$ does not change the $\mathfrak{g} \otimes \mathbb{C}[t]$ module structure because of the fusion filtration. Because of the filtration (12), this is still true if one adds a linear combination of $w_{j}$ with $j>i$. Thus we conclude that we can replace each vector $w_{i}$ by an arbitrary linear combination. In particular, there exist numbers $\alpha_{i, j}$ such that

$$
\sum_{j=1}^{N} \alpha_{i-1, j} w_{i}=v_{0}^{\otimes i} \otimes v_{1} \otimes v_{0}^{\otimes N-1-i} .
$$

Since $v_{1}$ is a highest weight vector of the adjoint representation of $\mathfrak{g}$ and $v_{0}$ spans the trivial representation, we arrive at the fact that

$$
\tilde{W}^{i}(1) / \tilde{W}^{i+1}(1) \simeq \mathfrak{g}^{* * N-1} .
$$

Because of the isomorphism (13), the $m=1$ case of the proposition is proved.

We are now going to connect the filtrations $G^{\bullet}(N)$ and the induced PBW filtration $F_{\bullet}(N)$ on the Demazure modules $D(N)$. We use Proposition 1 for $V_{i}=D(1)$.

Lemma 4. $G^{m}$ is a subspace of $F_{N-m}(N)$.

Proof. We first note that for the cyclic vector $v_{N} \in D(N)$ we have

$$
v_{N}=\left(e_{\theta} \otimes t^{-N}\right)^{N} v_{0} .
$$

Therefore, $G^{0}=F_{N}(N)=D(N)$ and our Lemma is true for $m=0$.

In general, we need to prove that

$$
\left(x_{1} \otimes t^{N+i_{1}}\right) \cdots\left(x_{m} \otimes t^{N+i_{m}}\right) v \in F_{N-m}
$$

for any $v \in D(N)$ and $x_{1}, \ldots, x_{m} \in \mathfrak{g}, i_{1}, \ldots, i_{m} \geq 0$. Since $v \in D(N)$ there exists an element $r \in \mathrm{U}(\mathfrak{g} \otimes \mathbb{C}[t])$ such that $v=r v_{N}$. Because of (14), the inclusion (15) follows from the following statement:

$$
\left(x_{1} \otimes t^{N+i_{1}}\right) \cdots\left(x_{m} \otimes t^{N+i_{m}}\right)\left(y_{1} \otimes t^{j_{1}}\right) \cdots\left(y_{m} \otimes t^{j_{m}}\right)\left(e_{\theta} \otimes t^{-N}\right)^{N} v_{0} \in F_{N-m}
$$

for arbitrary $x_{\alpha}, y_{\beta} \in \mathfrak{g}$ and $i_{\alpha}, j_{\beta}, n \geq 0$. Since $(\mathfrak{g} \otimes \mathbb{C}[t]) \cdot v_{0}=0$ the expression

$$
\left(y_{1} \otimes t^{j_{1}}\right) \cdots\left(y_{n} \otimes t^{j_{n}}\right)\left(e_{\theta} \otimes t^{-N}\right)^{N} v_{0}
$$

is equal to a linear combination of the vectors of the form

$$
\left(z_{1} \otimes t^{-N+l_{1}}\right) \cdots\left(z_{N} \otimes t^{-N+l_{N}}\right) v_{0}
$$

where $z_{i} \in \mathfrak{g}$ and $l_{i} \geq 0$. One can easily see that because of the condition $(\mathfrak{g} \otimes \mathbb{C}[t]) v_{0}=0$ the expression of the form

$$
\left(x \otimes t^{N+i}\right)\left(z_{1} \otimes t^{-N+l_{1}}\right) \cdots\left(z_{N} \otimes t^{-N+l_{N}}\right) v_{0}
$$

can be rewritten as a linear combination of the monomials of the form

$$
\left(z_{1} \otimes t^{-N+l_{1}}\right) \cdots\left(z_{N-1} \otimes t^{-N+1+l_{N-1}}\right) v_{0} .
$$

Iterating this procedure we arrive at (16). This proves (15) and hence our lemma is proved. 
Recall a notation for $q$-binomial coefficients

$$
\left(\begin{array}{c}
n \\
m
\end{array}\right)_{q}=\frac{(q)_{n}}{(q)_{m}(q)_{n-m}}, \quad(q)_{a}=(1-q) \cdots\left(1-q^{a}\right)
$$

For two $q$ series $f(q)=\sum f_{n} q^{n}$ and $g(q)=\sum g_{n} q^{n}$ we write $f \geq g$ if $f_{n} \geq g_{n}$ for all $n$.

Corollary 3. The following character inequality holds:

$$
\operatorname{ch}_{q} \operatorname{gr}_{m} F_{\bullet}(N) \geq q^{m^{2}}\left(\begin{array}{l}
N \\
m
\end{array}\right)_{q} \overline{\operatorname{ch}}_{q^{-1}} \mathfrak{g}^{* * m} .
$$

Proof. Because of the Lemma above, it suffices to show that

$$
\operatorname{ch}_{q} G^{m} / G^{m+1}=q^{(N-m)^{2}}\left(\begin{array}{l}
N \\
m
\end{array}\right) \overline{q h}_{q^{-1}} \mathfrak{g}^{* * N-m} .
$$

Note that $d v_{N}=N^{2} v_{N}$. Therefore, the graded character of the space of cyclic vectors $w_{i_{1}, \ldots, i_{m}}$ from Proposition 2 (where the $q$-degree of $u$ is fixed to be equal to $N$ according to Proposi-

tion 1) is given by $q^{m^{2}}\left(\begin{array}{l}N \\ m\end{array}\right)_{q}$. Multiplying by the $\bar{d}$ character of $\mathfrak{g}^{* * N-m}$ with respect to $\bar{d}$ (see Remark 3), we arrive at our Corollary.

Corollary 4. The following character inequality holds:

$$
\operatorname{ch}_{q} \operatorname{gr}_{m} F_{\bullet} \geq q^{m^{2}} \frac{1}{(q)_{m}} \overline{\operatorname{ch}}_{q^{-1}} \mathfrak{g}^{* * m} .
$$

Proof. Follows from limit formulas

$$
\lim _{N \rightarrow \infty} D(N)=L, \quad \lim _{N \rightarrow \infty}\left(\begin{array}{l}
N \\
m
\end{array}\right)_{q}=\frac{1}{(q)_{m}} .
$$

Remark 5. In the next section we prove that (18) is an equality. In Section 6 we prove that (17) is also an equality.

\section{Dual functional realization}

We now consider the restricted dual space to the "expected" PBW filtered space $L^{\text {gr }}$. Let

$$
\mathrm{U}^{a b}=\mathbb{C}\left[\mathfrak{g}^{a b} \otimes t^{-1} \mathbb{C}\left[t^{-1}\right]\right]
$$

be a space of polynomial functions on the space $\mathfrak{g}^{a b} \otimes t^{-1} \mathbb{C}\left[t^{-1}\right]$ (recall that $\mathfrak{g}^{a b}$ is an Abelian Lie algebra with the underlying vector space isomorphic to $\mathfrak{g}$ ). The algebra $\mathrm{U}^{a b}$ is an abelinization of the universal enveloping algebra $\mathrm{U}\left(\mathfrak{g} \otimes t^{-1} \mathbb{C}\left[t^{-1}\right]\right)$ of the Lie algebra of generating operators (due to the PBW theorem). We note that $\mathfrak{g}$ acts on the space $\mathfrak{g}^{a b} \otimes t^{-1} \mathbb{C}\left[t^{-1}\right]$ via the adjoint representation on $\mathfrak{g}^{a b}$.

Let $I \in \mathrm{U}^{a b}$ be the minimal $\mathfrak{g}$ invariant ideal, which contains all coefficients of $e^{\theta}(z)^{2}$, i.e. all elements of the form

$$
\sum_{1 \leq i \leq n}\left(e_{\theta} \otimes t^{-i}\right)\left(e_{\theta} \otimes t^{-n-1+i}\right), \quad n=1,2, \ldots
$$

Denote

$$
L^{\prime}=\mathrm{U}^{a b} / I \text {. }
$$


This space can be decomposed according to the number of variables in a monomial:

$$
L^{\prime}=\bigoplus_{m=0}^{\infty} L_{m}^{\prime}=\bigoplus_{m=0}^{\infty} \operatorname{span}\left\{x_{1} \otimes t^{i_{1}} \cdots x_{m} \otimes t^{i_{m}}, x_{i} \in \mathfrak{g}^{a b}\right\}
$$

The operator $d \in \widehat{\mathfrak{g}}$ induces a degree operator on $L^{\prime}$. We denote this operator by the same symbol. There exists a surjective homomorphism of $\mathfrak{g}^{a b} \otimes t^{-1} \mathbb{C}\left[t^{-1}\right]$ modules $L^{\prime} \rightarrow L^{\text {gr }}$ (see Lemma 2). Our goal is to show that $L^{\prime} \simeq L^{\text {gr }}$. Let $\left(L_{m}^{\prime}\right)^{*}$ be a restricted dual space:

$$
\left(L_{m}^{\prime}\right)^{*}=\bigoplus_{n \geq 0}\left(L_{m, n}^{\prime}\right)^{*}, \quad L_{m, n}^{\prime}=\left\{v \in L_{m}^{\prime}: d v=n v\right\}
$$

We construct the functional realization of $L_{m}^{\prime}$ using currents

$$
x(z)=\sum_{i>0}\left(x \otimes t^{-i}\right) z^{i}
$$

for $x \in \mathfrak{g}$. Following [13] we consider a map

$$
\alpha_{m}:\left(L_{m}^{\prime}\right)^{*} \rightarrow \mathbb{C}\left[z_{1}, \ldots, z_{m}\right] \otimes \mathfrak{g}^{\otimes m}, \quad \phi \mapsto r_{\phi}
$$

from the dual space $\left(L_{m}^{\prime}\right)^{*}$ to the space of polynomials in $m$ variables with values in the $m$-th tensor power of the space $\mathfrak{g}$. This map is given by the formula

$$
\left\langle r_{\phi}, x_{1} \otimes \cdots \otimes x_{m}\right\rangle=\phi\left(x_{1}\left(z_{1}\right) \cdots x_{m}\left(z_{m}\right)\right) \text {, }
$$

where brackets in the left hand side denote the product of non degenerate Killing forms on $n$ factors $\mathfrak{g}$. Our goal is to describe the image of $\alpha_{m}$. We first formulate the conditions on $r_{\phi}$, which follow from the definition of $L^{\prime}$ and then prove that these conditions determine the image of $\alpha_{m}$. We prepare some notations first.

Consider the decomposition of the tensor square $\mathfrak{g} \otimes \mathfrak{g}$ into the direct sum of $\mathfrak{g}$ modules:

$$
\mathfrak{g} \otimes \mathfrak{g}=V_{2 \theta} \oplus \bigwedge^{2} \mathfrak{g} \oplus S^{2} \mathfrak{g} / V_{2 \theta} .
$$

Here $V_{2 \theta}$ is a highest weight $\mathfrak{g}$-module with a highest weight $2 \theta$ embedded into $S^{2} \mathfrak{g}$ via the map

$$
V_{2 \theta} \simeq \mathrm{U}(\mathfrak{g}) \cdot\left(e_{\theta} \otimes e_{\theta}\right) \hookrightarrow S^{2} \mathfrak{g} .
$$

Lemma 5. For the module $\mathfrak{g} * * \mathfrak{g}$ we have

$$
\operatorname{gr}_{0}(\mathfrak{g} * * \mathfrak{g})=V_{2 \theta}, \quad \operatorname{gr}_{1}(\mathfrak{g} * * \mathfrak{g})=\bigwedge^{2} \mathfrak{g}, \quad \operatorname{gr}_{2}(\mathfrak{g} * * \mathfrak{g})=S^{2} \mathfrak{g} / V_{2 \theta}
$$

and all other graded components vanish.

Proof. We first show that $\operatorname{gr}_{n}(\mathfrak{g} * * \mathfrak{g})=0$ for $n>2$. Let $c_{1}, c_{2}$ be evaluation constants which appear in Definition 2 of the fusion product. Recall that $\mathfrak{g} * * \mathfrak{g}$ is independent of the evaluation parameters. So we can set $c_{1}=1, c_{2}=0$. Then the second space of the fusion filtration is given by

$$
\mathrm{U}(\mathfrak{g}) \cdot\left(\operatorname{span}\left\{\left[x_{1},\left[x_{2}, e_{\theta}\right]\right], x_{1}, x_{2} \in \mathfrak{g}\right\} \otimes e_{\theta}\right),
$$

where $\mathrm{U}(\mathfrak{g})$ acts on the tensor product $\mathfrak{g} \otimes \mathfrak{g}$ diagonally. But $\left[f_{\theta},\left[f_{\theta}, e_{\theta}\right]\right]=-2 f_{\theta}$ and hence

$$
\operatorname{span}\left\{\left[x_{1},\left[x_{2}, e_{\theta}\right]\right], x_{1}, x_{2} \in \mathfrak{g}\right\}=\mathfrak{g}
$$


(since the left hand side is invariant with respect to the subalgebra of $\mathfrak{g}$ of annihilating operators and contains the lowest weight vector $f_{\theta}$ of the adjoint representation). Therefore, (20) coincides with $\mathfrak{g} \otimes \mathfrak{g}$.

We now compute three nontrivial graded components $\operatorname{gr}_{0}(\mathfrak{g} * * \mathfrak{g}), \operatorname{gr}_{1}(\mathfrak{g} * * \mathfrak{g})$ and $\operatorname{gr}_{2}(\mathfrak{g} * * \mathfrak{g})$. From the definition of the fusion filtration we have

$$
\operatorname{gr}_{0}(\mathfrak{g} * * \mathfrak{g})=\mathrm{U}(\mathfrak{g}) \cdot\left(e_{\theta} \otimes e_{\theta}\right) \simeq V(2 \theta)
$$

We now redefine the evaluation parameters by setting $c_{1}=1, c_{2}=-1$. Then the formula for the operators $x \otimes t$ acting on $\mathfrak{g} \otimes \mathfrak{g}$ is given by $x \otimes \operatorname{Id}-\operatorname{Id} \otimes x$. These operators map $S^{2}(\mathfrak{g})$ to $\bigwedge^{2} \mathfrak{g}$ and vice versa. We conclude that

$$
\operatorname{gr}_{1}(\mathfrak{g} * * \mathfrak{g}) \hookrightarrow \bigwedge^{2} \mathfrak{g}, \quad \operatorname{gr}_{2}(\mathfrak{g} * * \mathfrak{g}) \hookrightarrow S^{2}(\mathfrak{g})
$$

Now our Lemma follows from the equality $\operatorname{gr}_{n}(\mathfrak{g} * * \mathfrak{g})=0$ for $n>2$.

Lemma 6. For any $\phi \in\left(L_{m}^{\prime}\right)^{*}$ the image $r_{\phi}$ is divisible by the product $z_{1} \cdots z_{m}$ and satisfies the vanishing condition

$$
\left\langle r_{\phi}, V_{2 \theta} \otimes \mathfrak{g}^{\otimes m-2}\right\rangle_{z_{1}=z_{2}}=0
$$

and the symmetry condition

$$
\sigma r=r, \quad \sigma \in S_{m}
$$

where the permutation group $S_{m}$ acts simultaneously on the set of variables $z_{1}, \ldots, z_{m}$ and on the tensor product $\mathfrak{g}^{\otimes m}$.

Proof. The product $z_{1} \cdots z_{m}$ comes from the condition that the highest weight vector is annihilated by $\mathfrak{g} \otimes \mathbb{C}[t]$, so for any $x \in \mathfrak{g}$ the series $x(z)$ starts with $z$. The condition (21) follows from the relation $e_{\theta}(z)^{2}=0$ and $\mathfrak{g}$-invariance of the ideal $I$. The symmetry condition follows from the commutativity of the algebra $\mathrm{U}^{a b}$.

We denote by

$$
V_{m} \hookrightarrow z_{1} \cdots z_{m} \mathbb{C}\left[z_{1}, \ldots, z_{m}\right] \otimes \mathfrak{g}^{\otimes m}
$$

the space of functions satisfying conditions (21) and (22). In the following lemma we endow $V_{m}$ with structures of representation of the ring of symmetric polynomials

$$
P_{m}^{\mathrm{sym}}=\mathbb{C}\left[z_{1}, \ldots, z_{m}\right]^{S_{m}}
$$

and of the current algebra $\mathfrak{g} \otimes \mathbb{C}[t]$.

Lemma 7. There exists natural structures of representations of $P_{m}^{\mathrm{sym}}$ and of $\mathfrak{g} \otimes \mathbb{C}[t]$ on $V_{m}$ defined by the following rule:

- $P_{m}^{\mathrm{sym}}$ acts on $V_{m}$ by multiplication on the first tensor factor.

- Lie algebra $\mathfrak{g} \otimes \mathbb{C}[t]$ acts on $V_{m}$ by the formula

$$
x \otimes t^{k} \quad \text { acts as } \sum_{i=1}^{n} z_{i}^{k} \otimes x^{(i)} .
$$

The actions of $P_{m}^{\mathrm{sym}}$ and of $\mathfrak{g} \otimes \mathbb{C}[t]$ commute. 
Proof. A direct computation.

Lemma 7 gives a structure of $\mathfrak{g} \otimes \mathbb{C}[t]$ module on the quotient space $V_{m} / P_{m+}^{\mathrm{sym}} V_{m}$, where the subscript + denotes the space of polynomials of positive degree. We will show that the dual to this module is isomorphic to $\mathfrak{g}^{* * m}$. We first consider the $m=2$ case.

Lemma 8. We have an isomorphism of representations of $\mathfrak{g} \otimes \mathbb{C}[t]$

$$
\left(V_{2} / P_{+}^{\mathrm{sym}} V_{2}\right)^{*} \simeq \mathfrak{g} * * \mathfrak{g} .
$$

Proof. Let $r$ be an element of $V_{2}$. Using the decomposition (19), we write $r=r_{0}+r_{1}+r_{2}$, where

$$
\begin{aligned}
& r_{0} \in z_{1} z_{2} \mathbb{C}\left[z_{1}, z_{2}\right] \otimes V_{2 \theta}, \\
& r_{1} \in z_{1} z_{2} \mathbb{C}\left[z_{1}, z_{2}\right] \otimes \Lambda^{2} \mathfrak{g}, \\
& r_{2} \in z_{1} z_{2} \mathbb{C}\left[z_{1}, z_{2}\right] \otimes S^{2} \mathfrak{g} / V_{2 \theta} .
\end{aligned}
$$

Then the conditions (21) and (22) are equivalent to

$$
\begin{aligned}
& r_{0} \in z_{1} z_{2}\left(z_{1}-z_{2}\right)^{2} P^{\mathrm{sym}} \otimes V_{2 \theta}, \\
& r_{1} \in z_{1} z_{2}\left(z_{1}-z_{2}\right) P^{\mathrm{sym}} \otimes \Lambda^{2} \mathfrak{g}, \\
& r_{2} \in z_{1} z_{2} P^{\mathrm{sym}} \otimes S^{2} \mathfrak{g} / V_{2 \theta} .
\end{aligned}
$$

Therefore, the dual quotient space $\left(V_{2} / P_{+}^{\mathrm{sym}} V_{2}\right)^{*}$ is isomorphic to $\mathfrak{g} * * \mathfrak{g}$ as a vector space. It is straightforward to check that the $\mathfrak{g} \otimes \mathbb{C}[t]$ modules structures are also isomorphic.

We will also need a nonsymmetric version of the space $V_{m} / P_{m+}^{\mathrm{sym}} V_{m}$. Namely, let $W_{m}$ be a subspace of $z_{1} \cdots z_{m} \mathbb{C}\left[z_{1}, \ldots, z_{m}\right] \otimes \mathfrak{g}^{\otimes m}$ satisfying

$$
\begin{aligned}
& \left.F_{z_{i}=z_{j}} \hookrightarrow \mathbb{C}\left[z_{1}, \ldots, z_{m}\right]\right|_{z_{1}=z_{2}} \otimes \mu_{i, j}\left(S^{2} \mathfrak{g} / V_{2 \theta} \otimes \mathfrak{g}^{\otimes m-2}\right), \\
& \left.\partial_{z_{i}} F_{z_{i}=z_{j}} \hookrightarrow \mathbb{C}\left[z_{1}, \ldots, z_{m}\right]\right|_{z_{1}=z_{2}} \otimes \mu_{i, j}\left(S^{2}(\mathfrak{g}) / V_{2 \theta} \otimes \mathfrak{g}^{\otimes m-2}\right)
\end{aligned}
$$

for all $1 \leq i<j \leq m$, where $\mu_{i, j}$ is an operator on the tensor power $\mathfrak{g}^{\otimes m}$ which inserts the first two factors on the $i$-th and $j$-th places respectively:

$$
\mu_{i, j}\left(v_{1} \otimes v_{2} \otimes v_{2} \otimes \cdots \otimes v_{n}\right)=v_{3} \otimes \cdots \otimes v_{i-1} \otimes v_{1} \otimes v_{i} \otimes \cdots \otimes v_{j-2} \otimes v_{2} \otimes v_{j-1} \cdots \otimes v_{n} .
$$

The natural action of the polynomial ring $P_{m}=\mathbb{C}\left[z_{1}, \ldots, z_{m}\right]$ commutes with the action of the current algebra, defined as in Lemma 7. Therefore, we obtain a structure of $\mathfrak{g} \otimes \mathbb{C}[t]$ module on the quotient space $W_{m} / P_{m+} W_{m}$, where $P_{m+}$ is the ring of positive degree polynomials. As proved in [13], Lemma 5.8, the symmetric and nonsymmetric constructions produce the same module (see Proposition 3 below for the precise statement). We illustrate this in the case $n=2$.

Lemma 9. $\left(W_{2} / P_{2+} W_{2}\right)^{*} \simeq \mathfrak{g} * * \mathfrak{g}$.

Proof. We use the decomposition $r=r_{0}+r_{1}+r_{2}$ as in Lemma 8 for $r \in W_{m}$. The conditions (23) and (24) are equivalent to the following conditions on $r_{i}$ :

$$
\begin{aligned}
& r_{0} \in\left(z_{1}-z_{2}\right)^{2} z_{1} z_{2} \mathbb{C}\left[z_{1}, z_{2}\right] \otimes V_{2 \theta}, \\
& r_{1} \in\left(z_{1}-z_{2}\right) z_{1} z_{2} \mathbb{C}\left[z_{1}, z_{2}\right] \otimes \Lambda^{2} \mathfrak{g}, \\
& r_{2} \in z_{1} z_{2} \mathbb{C}\left[z_{1}, z_{2}\right] \otimes S^{2} \mathfrak{g} / V_{2 \theta} .
\end{aligned}
$$

After passing to the quotient with respect to the action of the algebra $P_{2+}$ we arrive at the isomorphism of vector spaces $\left(W_{2} / P_{2+} W_{2}\right)^{*} \simeq \mathfrak{g} * \mathfrak{g}$. It is straightforward to check that this is an isomorphism of $\mathfrak{g} \otimes \mathbb{C}[t]$ modules. 
The following Proposition is proved in [13, Lemma 5.8] for $\mathfrak{g}=\mathfrak{s l}_{2}$.

Proposition 3. There exists an isomorphism of $\mathfrak{g} \otimes \mathbb{C}[t]$-modules

$$
V_{m} / P_{m+}^{\mathrm{sym}} V_{m} \simeq W_{m} / P_{m+} W_{m} .
$$

Proof. The proof of the general case differs from the one from [13] by the replacement of the decomposition of the tensor square of the adjoint representation of $\mathfrak{s l}_{2}$ by the general decomposition (19).

Remark 6. We note that the definition of the space $W_{m}$ is a bit more involved than the definition of $V_{m}$. The reason is that the polynomials used to construct $W_{m}$ are not symmetric. In particular, this forces to add the condition (24) in order to get the isomorphism $V_{m} / P_{m+}^{\mathrm{sym}} V_{m} \simeq$ $W_{m} / P_{m+} W_{m}$.

Proposition 4. The module $W_{m} / P_{+} W_{m}$ is cocyclic with a cocyclic vector being the class of

$$
r_{m}=z_{1} \cdots z_{m} \prod_{1 \leq i<j \leq m}\left(z_{i}-z_{j}\right)^{2} \otimes\left(e_{\theta}\right)^{\otimes m} .
$$

Proof. We first show that if $r \in W_{m}$ is a nonzero element satisfying $r \in V_{m \theta} \otimes \mathbb{C}\left[z_{1}, \ldots, z_{m}\right]$ then either $r \in P_{m+} W_{m}$ or there exists an element of the universal enveloping algebra of $\mathfrak{g} \otimes \mathbb{C}[t]$ which sends $r$ to $r_{m}$ (here we embed $V_{m \theta}$ into $\mathfrak{g}^{\otimes m}$ as an irreducible component containing $\left(e_{\theta}\right)^{\otimes m}$ ). In fact, from conditions (23), (24) and the assumption $r \in V_{m \theta} \otimes \mathbb{C}\left[z_{1}, \ldots, z_{m}\right]$ we obtain that $r$ is divisible by the product $\prod_{1 \leq i<j \leq m}\left(z_{i}-z_{j}\right)^{2}$. If $r \notin P_{m+} W_{m}$ then we obtain

$$
r=x \otimes \prod_{1 \leq i<j \leq m}\left(z_{i}-z_{j}\right)^{2}
$$

with some $x \in V_{m \theta}$. Since $V_{m \theta}$ is irreducible the $\mathrm{U}(\mathfrak{g})$ orbit of $x$ contains $\left(e_{\theta}\right)^{\otimes m}$.

Thus it suffices to show that any element $r \in W_{m} / P_{+} W_{m}$ is contained in the $\mathrm{U}(\mathfrak{g} \otimes \mathbb{C}[t])$ orbit of the image of $V_{m \theta} \otimes \mathbb{C}\left[z_{1}, \ldots, z_{m}\right]$ in the quotient space. We prove that if $(a \otimes t) r=0$ for all $a \in \mathfrak{g}$ then $r \in V_{m \theta} \otimes P_{m}$. This would imply the previous statement since $W_{m} / P_{m+} W_{m}$ is finite-dimensional.

So let $r \in W_{m}$ be some element and $\bar{r} \in W_{m} / P_{m+} W_{m}$ be its class. Assume that $(a \otimes t) r=0$ for all $a \in \mathfrak{g}$. We first show that $r \in V_{2 \theta} \otimes \mathfrak{g}^{\otimes n-2} \otimes P_{m}$. In fact, there exists a polynomial $l \in P_{m}$ such that the following holds in $W_{m}$ :

$$
(a \otimes t) r=l\left(z_{1}, \ldots, z_{m}\right) r_{1}
$$

for some $r_{1} \in W_{m}$. Consider the space of functions $W_{m}^{1,2}$ which satisfy conditions (23) and (24) only for $i=1, j=2$. (In particular, $W_{m} \hookrightarrow W_{m}^{1,2}$ ). We have an isomorphism of $\mathfrak{g} \otimes \mathbb{C}[t]$ modules

$$
W_{m}^{1,2} \simeq W_{2} \otimes \mathfrak{g}^{\otimes m-2} \otimes \mathbb{C}\left[z_{3}, \ldots, z_{m}\right] .
$$

The equality $(a \otimes t) r=l r_{1}$ gives

$$
\left(a^{(1)} \otimes z_{1}+a^{(2)} \otimes z_{2}\right) r=l r_{1}-\left(\sum_{i=3}^{m} a^{(i)} \otimes z_{i}\right) r .
$$

Using Lemma 9, we obtain $r \in V_{2 \theta} \otimes \mathfrak{g}^{\otimes n-2} \otimes P_{m}$.

The same procedure can be done for all pairs $1 \leq i<j \leq n$. Now our proposition follows from the following equality in $\mathfrak{g}^{\otimes n}$ :

$$
\bigcap_{1 \leq i<j \leq n} \mu_{i, j} V_{2 \theta} \otimes \mathfrak{g}^{\otimes n-2}=V_{n \theta}
$$


The dual module $\left(W_{m} / P_{m} W_{m}\right)^{*}$ is cyclic. We denote by $r_{m}^{\prime}$ a cyclic vector which corresponds to the cocyclic vector $r_{m}$.

Proposition 5. There exists a surjective homomorphism of $\mathfrak{g} \otimes \mathbb{C}[t]$ modules

$$
\mathfrak{g}^{* * m} \rightarrow\left(W_{m} / P_{m+} W_{m}\right)^{*}
$$

sending a cyclic vector $e_{\theta}^{\otimes m}$ to $r_{m}^{\prime}$.

Proof. A relation in the fusion product means that some expression of the form

$$
\sum \alpha_{i_{1} \ldots i_{s}}\left(x_{1} \otimes t^{i_{1}}\right) \cdots\left(x_{s} \otimes t^{i_{s}}\right)
$$

with fixed $t$ degree can be expressed in $\mathfrak{g}^{\otimes m}$ via a linear combination of monomials of lower $t$-degree. The coefficients of the expression of (26) in terms of the lower degree monomials are polynomials in evaluation parameters $c_{1}, \ldots, c_{n}$. Therefore, by the very definition of the action of $P_{m}$ on $W_{m}$, the operator of the form (26), which is a relation in the fusion product, vanishes on $\left(W_{m} / P_{m+} W_{m}\right)^{*}$.

Note that $W_{m}$ and $V_{m}$ are naturally graded by the degree grading on $P_{m}\left(P_{m}^{\mathrm{sym}}\right)$. This grading defines a graded character of the space $V_{m} / P_{m+}^{\text {sym }}$.

Corollary 5. $\operatorname{ch}_{q} V_{m} / P_{m+} V_{m} \leq q^{m^{2}} \overline{c h}_{q^{-1}} \mathfrak{g}^{* * m}$.

Proof. Follows from Proposition 5 and Proposition 3. Note that the factor $m^{2}$ is a degree of the cyclic vector $r_{m}^{\prime}$.

Remark 7. In the next section we combine Corollary 5 with Corollary 4 in order to compute the character of $L^{\mathrm{gr}}$.

\section{Proofs of the main statements}

Proposition 6. $\operatorname{ch}_{q}\left(L_{m}\right)^{*} \leq \operatorname{ch}_{q} V_{m} \leq q^{m^{2}} \overline{\operatorname{ch}}_{q^{-1}} \mathfrak{g}^{* * m} /(q)_{m}$.

Proof. We know that

$$
\operatorname{ch}_{q} L_{m}^{*} \leq \operatorname{ch}\left(L_{m}^{\prime}\right)^{*}=\operatorname{ch}_{q} V_{m} .
$$

Because of the surjection (25), the character of $V_{m}^{*}$ is smaller than or equal to $q^{m^{2}} \overline{\operatorname{ch}}_{q^{-1}} \mathfrak{g}^{* * m} /(q)_{m}$ (since $1 /(q)_{m}$ is the character of the space of symmetric polynomials in $m$ variables). This proves the Proposition.

Theorem 4. $L_{m} \simeq L_{m}^{\prime}$.

Proof. Corollary 4 provides an inequality

$$
\operatorname{ch}_{q} L_{m} \geq \frac{q^{m^{2}}}{(q)_{m}} \overline{\operatorname{ch}}_{q^{-1}} \mathfrak{g}^{* * m} .
$$

Now from Proposition 6 we obtain

$$
\frac{q^{m^{2}}}{(q)_{m}} \overline{\operatorname{ch}}_{q^{-1}} \mathfrak{g}^{* * m} \leq \operatorname{ch}_{q} L_{m} \leq \operatorname{ch}_{q} L_{m}^{\prime} \leq \frac{q^{m^{2}}}{(q)_{m}} \overline{\operatorname{ch}}_{q^{-1}} \mathfrak{g}^{* * m} .
$$

Theorem is proved. 
Corollary 6. The dual module $\left(V_{m} / P_{m+}^{\mathrm{sym}} V_{m}\right)^{*}$ and $\mathfrak{g}^{* * m}$ are isomorphic as $\mathfrak{g} \otimes \mathbb{C}[t]$ modules.

Proof. Follows from Propositions 5, 3 and Theorem 4.

Corollary 7. We have an isomorphism of $\mathfrak{g}^{a b} \otimes \mathbb{C}\left[t^{-1}\right]$ modules

$$
L^{\mathrm{gr}} \simeq \mathrm{U}\left(\mathfrak{g}^{a b} \otimes \mathbb{C}\left[t^{-1}\right]\right) / I,
$$

where $I$ is the minimal $\mathfrak{g}$ invariant ideal containing the coefficients of the current $e_{\theta}(z)^{2}=0$.

Remark 8. Corollary 7 is a generalization of the $\mathfrak{s l}_{2}$ case from [13]. It also proves a level 1 conjecture from [10].

Corollary 8. The action of the polynomial ring $P_{m}^{\mathrm{sym}}$ on $V_{m}$ is free.

Proof. Follows from the isomorphism $\left(L_{m} / P_{m+}^{\mathrm{sym}}\right)^{*} \simeq \mathfrak{g}^{* * m}$ and the character equality

$$
\operatorname{ch}_{q} L_{m}=\frac{q^{m^{2}}}{(q)_{m}} \overline{\operatorname{ch}}_{q^{-1}} \mathfrak{g}^{* * m}=q^{m^{2}} \operatorname{ch}_{q} P_{m}^{\mathrm{sym}} \cdot \overline{\operatorname{ch}}_{q^{-1}} \mathfrak{g}^{* * m} .
$$

Recall the vectors

$$
w_{i_{1}, \ldots, i_{m}}=\left(e_{\theta} \otimes t^{i_{1}}\right) \cdots\left(e_{\theta} \otimes t^{i_{m}}\right) v_{0} \in L .
$$

Let $\bar{w}_{i_{1}, \ldots, i_{m}} \in L_{m}$ be the images of these vectors.

Corollary 9. $L_{m}$ is generated by the set of vectors

$$
\bar{w}_{i_{1}, \ldots, i_{m}}, \quad-m \geq i_{1} \geq \cdots \geq i_{m}
$$

with the action of the algebra $\mathrm{U}(\mathfrak{g} \otimes \mathbb{C}[t])$.

Proof. Recall the element

$$
r_{m}=z_{1} \cdots z_{m} \prod_{1 \leq i<j \leq m}\left(z_{i}-z_{j}\right)^{2} \otimes e_{\theta}^{\otimes N} \in V_{m} .
$$

Since $r_{m}$ is a cocyclic vector of $V_{m} / P_{m+}^{\text {sym }}$ and the polynomial algebra acts freely on $V_{m}$, we obtain that the space $P_{m+}^{\mathrm{sym}} r_{m}$ is a cocyclic subspace of $V_{m}$, which means that for any vector $v \in V_{m}$ the space $\mathrm{U}(\mathfrak{g} \otimes \mathbb{C}[t]) \cdot v$ has a nontrivial intersection with $P_{m+}^{\mathrm{sym}} r_{m}$. We note that $P_{m+}^{\mathrm{sym}} r_{m}$ coincides with the subspace of $V_{m}$ of $\mathfrak{g}$ weight $m \theta$. Dualizing this construction we obtain that the subspace of $L_{m}$ of $\mathfrak{g}$ weight $-m \theta$ is $\mathrm{U}(\mathfrak{g} \otimes \mathbb{C}[t])$ cyclic. This space is linearly spanned by the set (27). Corollary is proved.

Corollary 10. The space $\operatorname{gr}_{m} F_{\bullet}(N)$ is generated by the set of vectors

$$
\bar{w}_{i_{1}, \ldots, i_{m}}, \quad-m \geq i_{1} \geq \cdots \geq i_{m} \geq-N
$$

with the action of the algebra $\mathrm{U}(\mathfrak{g} \otimes \mathbb{C}[t])$.

Proof. Introduce an increasing filtration $J_{\bullet}$ on $L_{m}$ :

$$
J_{n}=\mathrm{U}(\mathfrak{g} \otimes \mathbb{C}[t]) \cdot \operatorname{span}\left\{\bar{w}_{i_{1}, \ldots, i_{m}}:-i_{1}-\cdots-i_{m} \leq m^{2}+n\right\} .
$$

Corollary 6 provides an isomorphism of $\mathfrak{g} \otimes \mathbb{C}[t]$ modules

$$
\mathrm{U}(\mathfrak{g} \otimes \mathbb{C}[t]) \cdot \bar{w}_{i_{1}, \ldots, i_{m}} /\left(J_{-i_{1}-\cdots-i_{m}-1} \cap \mathrm{U}(\mathfrak{g} \otimes \mathbb{C}[t]) \cdot \bar{w}_{i_{1}, \ldots, i_{m}}\right) \simeq \mathfrak{g}^{* * m} .
$$


Thus, since all vectors of the form $(28)$ belong to $F_{m}(N)$, we obtain

$$
\operatorname{dim} \operatorname{gr}_{m} F_{\bullet}(N) \geq\left(\begin{array}{l}
N \\
m
\end{array}\right)(\operatorname{dim} \mathfrak{g})^{m} .
$$

Because of the equality

$$
\operatorname{dim} D(N)=(1+\operatorname{dim} \mathfrak{g})^{N}=\sum_{m=0}^{N}\left(\begin{array}{l}
N \\
m
\end{array}\right)(\operatorname{dim} \mathfrak{g})^{m},
$$

we conclude that $\operatorname{dim} \operatorname{gr}_{m} F_{\bullet}(N)=\left(\begin{array}{l}N \\ m\end{array}\right)(\operatorname{dim} \mathfrak{g})^{m}$ and hence the whole space $\operatorname{gr}_{m} F_{\bullet}(N)$ is generated by the vectors $(28)$.

Proposition 7. The induced $P B W$ filtration $F_{\bullet}(N) \hookrightarrow D(N)$ coincides with the $t^{N}$-filtration $G^{\bullet}$, i.e. $F_{m}(N)=G^{N-m}$.

Proof. Recall (see Lemma 4) that $G^{N-m} \hookrightarrow F_{m}(N)$. Since $v_{0}$ is proportional to $\left(f_{\theta} \otimes t^{N}\right)^{N} v_{N}$, we obtain that $\bar{w}_{i_{1}, \ldots, i_{m}} \in G^{N-m}$ for $-m \geq i_{1} \geq \cdots \geq i_{m} \geq-N$. Therefore, Corollary 10 gives $F_{m}(N) \hookrightarrow G^{N-m}$. Proposition is proved.

Corollary 11. The graded component $\mathrm{gr}_{m} F_{\bullet}(N)$ is filtered by $\left(\begin{array}{l}N \\ m\end{array}\right)$ copies of $\mathfrak{g}^{* * m}$. The character of the space of cyclic vectors of those fusions is equal to $q^{m^{2}}\left(\begin{array}{l}N \\ m\end{array}\right)_{q}$.

We summarize all above in the following theorem:

Theorem 5. Let $F_{\bullet}$ be the PBW filtration on the level one vacuum $\widehat{\mathfrak{g}}$ module L. Then

a) $\operatorname{gr}_{m} F_{\bullet}$ is filtered by the fusion modules $\mathfrak{g}^{* * m}$.

b) The character of the space of cyclic vectors of these $\mathfrak{g}^{* * m}$ is equal to $\frac{q^{m^{2}}}{(q)_{m}}$.

c) The induced $P B W$ filtration on Demazure modules $D(N)$ coincides with the double fusion filtration coming from Theorem 3.

d) The defining relation in $L^{\mathrm{gr}}$ is $e_{\theta}(z)^{2}=0$.

\section{A list of the main notations}

$\mathfrak{g}$ - simple finite-dimensional algebra;

$\widehat{\mathfrak{g}}$ - corresponding affine Kac-Moody algebra;

$\theta$ - highest weight of the adjoint representation of $\mathfrak{g}$;

$e_{\theta}, f_{\theta} \in \mathfrak{g}-$ highest and lowest weight vectors of the adjoint representation;

$L$ - the basic (vacuum level one) representation of $\widehat{\mathfrak{g}}$;

$v_{0} \in L-$ a highest weight vector of $L$;

$v_{N} \in L-$ an extremal vector of the weight $N \theta$;

$D(N) \hookrightarrow L-$ Demazure module with a cyclic vector $v_{N}$;

$\operatorname{gr}_{m} A_{\bullet}-m$-th graded component of the associated graded space with respect to the filtration $A_{\bullet}$;

$F_{\bullet}$ - (increasing) PBW filtration on $L$; 
$F_{\bullet}(N)=F_{\bullet} \cap D(N)$ - induced PBW filtration on $D(N)$;

$G^{\bullet}-($ decreasing $) t^{N}$-filtration on $D(N)$;

$V_{1} * \cdots * V_{N}$ - associated graded $\mathfrak{g} \otimes \mathbb{C}[t]$ module with respect to the fusion filtration on the tensor product of cyclic $\mathfrak{g} \otimes \mathbb{C}[t]$ modules;

$V_{1} * * \cdots * * V_{N}-$ associated graded $\mathfrak{g} \otimes \mathbb{C}[t]$ module with respect to the fusion filtration on the tensor product of cyclic $\mathfrak{g}$ modules;

$\mathrm{gr}_{m}\left(V_{1} * \cdots * V_{N}\right)-m$-th graded component with respect to the fusion filtration;

$\mathrm{ch}_{q}$ - a graded character defined by the operator $d$;

$\overline{\mathrm{ch}}_{q}$ - a graded character defined by the operator $\bar{d}$.

\section{Acknowledgements}

EF thanks B. Feigin and P. Littelmann for useful discussions. This work was partially supported by the RFBR Grants 06-01-00037, 07-02-00799 and NSh-3472.2008.2, by Pierre Deligne fund based on his 2004 Balzan prize in mathematics, by Euler foundation and by Alexander von Humboldt Fellowship.

\section{References}

[1] Ardonne E., Kedem R., Fusion products of Kirillov-Reshetikhin modules and fermionic multiplicity formulas, J. Algebra 308 (2007), 270-294, math.RT/0602177.

[2] Ardonne E., Kedem R., Stone M., Fusion products, Kostka polynomials and fermionic characters of $\widehat{s u}(r+$ 1) $)_{k}$, J. Phys. A: Math. Gen. 38 (2005), 9183-9205, math-ph/0506071.

[3] Frenkel E., Ben-Zvi D., Vertex algebras and algebraic curves, Mathematical Surveys and Monographs, Vol. 88, Amer. Math. Soc., Providence, RI, 2001.

[4] Chari V., Le T., Representations of double affine Lie algebras, in A Tribute to C.S. Seshadri (Chennai, 2002), Trends Math., Birkhäuser, Basel, 2003, 199-219, math.QA/0205312.

[5] Chari V., Loktev S., Weyl, Demazure and fusion modules for the current algebra of $\mathfrak{s l}_{r+1}$, Adv. Math. 207 (2006), 928-960, math.QA/0502165.

[6] Chari V., Presley A., Weyl modules for classical and quantum affine algebras, Represent. Theory 5 (2001), 191-223, math.QA/0004174.

[7] Demazure M., Désingularisation des variétés de Schubert généralisées, Ann. Sci. École Norm. Sup. (4) 7 (1974), 53-88.

[8] Di Francesco P., Mathieu P., Sénéchal D., Conformal field theory, Springer-Verlag, New York, 1997.

[9] Feigin E., Infinite fusion products and $\widehat{\mathfrak{s l}}_{2}$ cosets, J. Lie Theory 17 (2007), 145-161, math.QA/0603226.

[10] Feigin E., The PBW filtration, math.QA/0702797.

[11] Feigin B., Feigin E., Q-characters of the tensor products in $\mathfrak{s l}_{2}$ case, Moscow Math. J. 2 (2002), 567-588, math.QA/0201111.

[12] Feigin B., Feigin E., Integrable $\widehat{\mathfrak{s l}_{2}}$-modules as infinite tensor products, in Fundamental Mathematics Today, Editors S. Lando and O. Sheinman, Independent University of Moscow, 2003, 304-334, math.QA/0205281.

[13] Feigin B., Feigin E., Jimbo M., Miwa T., Takeyama Y., A $\phi_{1,3}$-filtration on the Virasoro minimal series $M\left(p, p^{\prime}\right)$ with $1<p^{\prime} / p<2$, Publ. Res. Inst. Math. Sci. 44 (2008), 213-257, math.QA/0603070.

[14] Feigin B., Jimbo M., Kedem R., Loktev S., Miwa T., Spaces of coinvariants and fusion product. I. From equivalence theorem to Kostka polynomials, Duke. Math. J. 125 (2004), 549-588, math.QA/0205324.

Feigin B., Jimbo M., Kedem R., Loktev S., Miwa T., Spaces of coinvariants and fusion product. II. $\widehat{\mathfrak{s l}_{2}}$ character formulas in terms of Kostka polynomials, J. Algebra 279 (2004), 147-179, math.QA/0208156.

[15] Frenkel I.B., Kac V.G., Basic representations of affine Lie algebras and dual resonance models, Invent. Math. 62 (1980/1981), 23-66. 
[16] Feigin B., Kirillov A.N., Loktev S., Combinatorics and geometry of higher level Weyl modules, in Moscow Seminar on Mathematical Physics. II, Amer. Math. Soc. Transl. Ser. 2, Vol. 221, Amer. Math. Soc., Providence, RI, 2007, 33-47, math.QA/0503315.

[17] Feigin B., Loktev S., On generalized Kostka polynomials and quantum Verlinde rule, in Differential Topology, Infinite-Dimensional Lie Algebras and Applications, Amer. Math. Soc. Transl. Ser. 2, Vol. 194, Amer. Math. Soc., Providence, RI, 1999, 61-79, math.QA/9812093.

[18] Fourier G., Littelmann P., Tensor product structure of affine Demazure modules and limit constructions, Nagoya Math. J. 182 (2006), 171-198, math.RT/0412432.

[19] Fourier G., Littelmann P., Weyl modules, Demazure modules, KR-modules, crystals, fusion products and limit constructions, Adv. Math. 211 (2007), 566-593, math.RT/0509276.

[20] Hernandez D., Quantum toroidal algebras and their representations, Selecta Math., to appear, arXiv:0801.2397.

[21] Kac V., Infinite-dimensional Lie algebras, 3rd ed., Cambridge University Press, Cambridge, 1990.

[22] Kac V., Vertex algebras for begginers, University Lecture Series, Vol. 10. Amer. Math. Soc., Providence, RI, 1997.

[23] Kedem R., Fusion products, cohomology of $G L_{N}$ flag manifolds, and Kostka polynomials, Int. Math. Res. Not. 2004 (2004), no. 25, 1273-1298, math.RT/0312478.

[24] Kreiman V., Lakshmibai V., Magyar P., Weyman J., Standard bases for affine SL(n)-modules, Int. Math. Res. Not. 2005 (2005), no. 21, 1251-1276, math.RT/0411017.

[25] Kumar S., Kac-Moody groups, their flag varieties and representation theory, Progress in Mathematics, Vol. 204, Birkhäuser Boston, Inc., Boston, MA, 2002.

[26] Loktev S., Weight multiplicity polynomials of multi-variable Weyl modules, arXiv:0806.0170. 